CORRECTION

https://doi.org/10.1038/s41586-018-0231-y

\title{
Author Correction: Diffusible repression of cytokinin signalling produces endodermal symmetry and passage cells
}

Tonni Grube Andersen, Sadaf Naseer, Robertas Ursache, Brecht Wybouw, Wouter Smet, Bert De Rybel,

Joop E. M. Vermeer \& Niko Geldner

Correction to: Nature https://doi.org/10.1038/nature25976, published online 14 March 2018.

In this Letter, owing to a copying error in Illustrator, the two centre panels in Extended Data Fig. 7a were identical. This error has been corrected online. The old, incorrect Extended Data Fig. 7 is shown in the Supplementary Information to this Amendment for transparency. Some typos ('occurence') in Figs. 1, 2 and 3 have also been corrected and the publication details for ref. 32 have been added.

Supplementary Information is available for this Amendment at https://doi. org/10.1038/s41586-018-0231-y. 